\title{
On the choice of the shunt circuit for single-mode vibration damping of piezoactuated structures
}

\author{
Paolo Bisegna ${ }^{1}$, Giovanni Caruso ${ }^{2}$, and Franco Maceri ${ }^{1}$ \\ 1 University of Rome "Tor Vergata", 00133 Rome, Italy \\ 2 Institute for Technology in Construction, ITC-CNR, 00137 Rome, Italy
}

\begin{abstract}
This paper deals with single-mode passive damping of piezoactuated structures. The problem of shunting in the best way a piezoelectric actuator is discussed, and a new passive shunt circuit, given by the parallel of an inductance and a capacitance in series to a resistance, is here proposed. For a sufficiently high piezoelectric coupling coefficient, it is analytically shown to be more performant than the classical resistive-inductive shunt circuit, in the sense that it guarantees an higher exponential time decay rate of the free vibrations.
\end{abstract}

\section{Introduction}

Piezoelectric materials, due to their lightness and easy integrability to the host structure, have been intensely applied for vibration control in special applications, like aeronautic and aerospace structures. Different strategies to vibration control are available: passive, active or hybrid $[2,4]$. Passive vibration damping, which is dealt with in this paper, is intrinsically stable, cheap and easy to be implemented by using piezoelectric actuators. The actuators are bonded to the mechanical structure and shunted to suitable external electric circuits containing at least resistive components, able to dissipate electric energy $[7,5,6]$. The basic idea can be easily understood looking on a simple, linearly elastic strut equipped with a piezoelectric patch and vibrating along an eigenmode. Due to the piezoelectric effect, at the electrodes of the piezoelectric device, acting as a capacitor, an alternating voltage arises. It is quite a natural idea to connect the electrodes on a passive circuit, in order to obtain an alternating current and an energy dissipation. The simplest shunt is the resonant one, tuned on the same frequency of the motion to be damped, in order to obtain, at that frequency, the lowest, purely resistive impedance and the maximum current for the induced voltage. It appears that the basic requirement is to dissipate as much energy as possible in the lowest time, with a circuit easy to be implemented, stable and cheap. Another requirement may concern the performance of the shunt in a frequency band large enough to include different structural eigenmodes.

In [7] both a resistive $(R)$ and a resistive-inductive $(R L)$ shunt circuit applied to the vibration damping of a single-mode piezoactuated structure 
were thoroughly examined. A slight variation of the $R L$ series circuit, i.e. the $R L$ parallel circuit, was presented in [8]. Many authors were involved in the analysis of those circuits $[9,10]$ and some modifications were proposed in order to improve their performances [11-13].

A major problem arises from the values of the electrical parameters of the piezoelectric components commercially available, because the inductors needed to tune the resonant circuit at the mechanical frequencies of interest are usually very high. Consequently, some authors [3] were considering the use of synthetic inductors. Later, others synthetic components of special shunt circuits were proposed, including negative capacitances [14].

At the present time, the $R L$ series circuit is generally accepted as the most performant passive shunt in vibration damping on a single eigenmode. Aim of this paper is to show that different schemes can be proposed, which are more performant than the $R L$ circuit, for a sufficiently high piezoelectric coupling coefficient. This result is obtained by choosing as performance index, and therefore optimization criterium, the exponential time-decay rate (ETDR) of the free vibrations. The maximization of such an objective function can be obtained, due to the linearity of the differential model of the electromechanical system, by means of an application of the pole placement technique to the system transfer function.

The new passive shunt circuit presented here in order to outperform over the classical $R L$ shunt circuit, is indicated by $R(L \| C)$ and is shown in Fig. 1. It is composed by the parallel of an inductance $L$ and a capacitance $C$, in series to a resistance $R$.

The analysis is analytically carried out, and closed-form expressions for the optimal values of the electrical components and of the achieved ETDR are determined. This analysis turns out to be very useful for design purposes and effective comparisons with the classical $R L$ series shunt circuit, in terms of optimal values of circuit parameters and achieved ETDR.

\section{The electromechanical model}

A single-degree-of-freedom mechanical system equipped with a piezoelectric device shunted on a passive circuit is here considered. The governing equations, in the Laplace domain, are [1,13]:

$$
\begin{aligned}
& \left(s^{2} m+k_{m m}\right) x+k_{m e} V=s x_{o}+\dot{x}_{o} \\
& q=-k_{m e} x+C_{p} V \\
& V+s Z q=0
\end{aligned}
$$

In the domain of the Laplace variable $s, x$ is the mechanical variable, $V$ is the electric potential and $q$ is the electric charge on the piezoelectric electrode. Moreover, $m$ is the mass, $k_{m m}$ is the mechanical stiffness, $k_{m e}$ is the electro-mechanical coupling stiffness and $x_{o}, \dot{x}_{o}$ are the initial displacement 


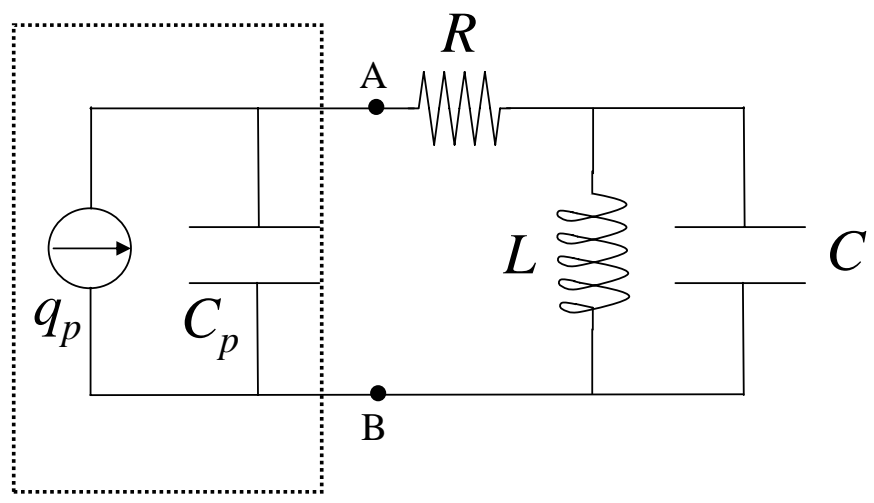

Piezoelectric element

Fig. 1. $R(L \| C)$ shunt circuit

and velocity. The electric initial conditions are assumed to vanish, for the sake of simplicity. Equation (1a) is the dynamic equilibrium of the mass $m$, equation (1b) is the free electric charge balance equation on the piezoelectric surfaces and equation (1c) is the Kirchhoff equation of the shunt electric circuit connected to the piezoelectric electrodes, whose impedance is denoted by $Z$.

In the case of a complex structure, equations (1a)-(1b) can be regarded as modal equations describing the evolution along a chosen eigenmode. Accordingly, the involved parameters can be evaluated by means of a suitable finite element formulation (e.g., [15]).

The shunt circuit described in the Introduction and reported in Fig. 1 is considered here. Its impedance is:

$$
Z=R+\frac{1}{(s L)^{-1}+s C}
$$

By substituting $V$ from equation (1b) into equations (1a) and (1c), and taking into account the above expression of $Z$, the governing equations are rewritten as follows:

$$
\begin{aligned}
& \left(s^{2} m+k_{m m}+\frac{k_{m e}^{2}}{C_{p}}\right) x+\frac{k_{m e}}{C_{p}} q=s x_{o}+\dot{x}_{o} \\
& \frac{k_{m e}}{C_{p}} x+\left(\frac{1}{C_{p}}+\frac{s R+s^{3} L R C+s^{2} L}{1+s^{2} L C}\right) q=0
\end{aligned}
$$

In order to study the system $(3 a)-(3 b)$ and to optimize the electrical parameters $R, L$ and $C$ in order to achieve the maximum ETDR, it is convenient to rewrite it in a dimensionless version. By letting $x=\bar{x} X, q=\bar{q} Q$ and $s=\bar{s} S$, where the capital letters mean dimensionless quantities and the 
overlined letters are the corresponding dimensional scales, a dimensionless version of $(3 a)-(3 b)$ can be written as:

$$
\begin{aligned}
& \left(S^{2}+1+\kappa^{2}\right) X+\kappa Q=s X_{o}+\dot{X}_{o} \\
& \kappa X+\frac{1+\rho S+\lambda(1+c) S^{2}+\lambda c \rho S^{3}}{1+c \lambda S^{2}} Q=0
\end{aligned}
$$

where the following dimensionless parameters have been introduced:

$$
\begin{array}{lll}
\kappa=\frac{k_{m e}}{\sqrt{k_{m m} C_{p}}}, & c=\frac{C}{C_{p}}, \quad & \rho=R \omega_{m} C_{p}, \quad \lambda=\omega_{m}^{2} C_{p} L, \\
\omega_{m}=\sqrt{\frac{k_{m m}}{m}}, & \frac{\bar{x}}{\bar{q}}=\sqrt{\frac{1}{k_{m m} C_{p}}}, \quad \bar{s}=\omega_{m}
\end{array}
$$

and $X_{o}, \dot{X}_{o}$ are the dimensionless counterparts of $x_{o}, \dot{x}_{o}$, respectively. In equation (5), $\kappa$ is the piezoelectric coupling coefficient and depends only on the piezoactuated structure, $c$ is the ratio between the capacity $C$ of the shunt circuit and the piezoelectric capacity $C_{p}, \lambda$ is the dimensionless inductance and $\rho$ is the dimensionless resistance.

\section{Optimization of the shunt circuit}

In this section an optimization of the dimensionless quantities $c, \lambda$ and $\rho$ is performed to maximize the exponential time decay rate of the solution. The characteristic polynomial $p(S)$ of the dimensionless system (4a)-(4b) reads explicitly as:

$$
\begin{gathered}
p(S)=S^{5}+\frac{1+c}{c \rho} S^{4}+\left(\frac{1}{c \lambda}+1+\kappa^{2}\right) S^{3} \\
+\frac{\lambda\left(1+\kappa^{2}\right)+\lambda c+1}{c \lambda \rho} S^{2}+\frac{1+\kappa^{2}}{c \lambda} S+\frac{1}{c \lambda \rho}
\end{gathered}
$$

The system poles $S_{i}$ are the roots of the above polynomial $p(S)$. They have a nonpositive real part, due to the inherent passivity of the system. The exponential time decay rate of the free vibrations is given by:

$$
\operatorname{ETDR}=-\max _{i}\left\{\operatorname{Re}\left(S_{i}\right)\right\}
$$

where Re denotes the real part of a complex number. Consequently, the optimization problem amounts to searching for the values of $\lambda, \rho$ and $c$ which maximize the ETDR for each fixed value of $\kappa$. It is here remarked that the poles of the dimensionless system $(4 \mathrm{a})-(4 \mathrm{~b})$ are proportional to the poles of (3a) $-(3 \mathrm{~b})$ trough the time-scale coefficient $\omega_{m}^{-1}$. 


\subsection{Analysis of the asymptotic case $c \rightarrow 0$}

In this section it is proved that the addition of a small capacitance in parallel to the inductance $L$ of a $R L$ series shunt circuit increases the ETDR, provided that the piezoelectric coupling coefficient is sufficiently high. To this end, an asymptotic analysis near $c=0$ is developed.

In the case $c=0$, the impedance $Z$ in equation (2) reduces $R+s L$. Consequently, the dimensionless system $(4 \mathrm{a})-(4 \mathrm{~b})$ reduces to the case of a $R L$ shunt circuit and the characteristic polynomial becomes $[7,13]$ :

$$
\bar{p}(S)=S^{4}+\frac{\rho}{\lambda} S^{3}+\left(1+\kappa^{2}+\frac{1}{\lambda}\right) S^{2}+\frac{\rho}{\lambda}\left(1+\kappa^{2}\right) S+\frac{1}{\lambda}
$$

which can be also obtained by taking the limit of $\operatorname{cop}(S)$ for $c \rightarrow 0$. Accordingly, the optimal values of $\lambda$ and $\rho$ can be computed, in the practical situation $0<\kappa<2$, by enforcing that $\bar{p}(S)$ has two coincident couples of complex conjugate roots:

$$
\bar{p}(S)=\left(S^{2}-2 x_{1} S+x_{1}^{2}+x_{2}^{2}\right)^{2}
$$

Here $x_{1}$ and $x_{2}$ are, respectively, the real and the imaginary part of the complex conjugate roots, and the ETDR coincides with $-x_{1}$. The optimal values of the parameters $\rho$ and $\lambda$ and the corresponding ETDR are $[1,13]$

$$
\lambda=\frac{1}{\left(1+\kappa^{2}\right)^{2}}, \quad \rho=\frac{2 \kappa}{\left(1+\kappa^{2}\right)^{\frac{3}{2}}}, \quad \mathrm{ETDR}=\frac{\kappa \sqrt{1+\kappa^{2}}}{2}
$$

Returning now to the case $c>0$ and limiting the analysis to the case of $c$ close to 0 , the following factorization of the fifth-order degree polynomial $p(S)$ is assumed:

$$
p(S)=\left(S^{2}-2 x_{1} S+x_{1}^{2}+x_{2}^{2}\right)^{2}\left(S-x_{3}\right)
$$

Here $x_{3}$ is a real root which approaches $-\infty$ when $c \rightarrow 0$ : hence, for each fixed small value of $c$, the ETDR is still the opposite of $x_{1}$.

By identifying the expressions (6) and (11) of $p(S)$, five nonlinear equations in the six unknowns $x_{1}, x_{2}, x_{3}, \lambda, \rho$ and $c$ are obtained, where $\kappa$ is intended to be kept fixed. By an elimination procedure, a single nonlinear equation $f\left(x_{1}, c\right)=0$ is obtained, which implicitly defines a function $x_{1}(c)$. The derivative $\partial x_{1} / \partial c$ can be obtained by a straightforward application of Dini's theorem. In particular, for $c=0$ this derivative is given by

$$
\left.\frac{\partial x_{1}(c)}{\partial c}\right|_{c=0}=-\frac{\kappa}{4} \frac{2 \kappa^{2}-1}{\sqrt{1+\kappa^{2}}}
$$

and is plotted versus $\kappa$ in Fig. 2 .

It is emphasized that $\partial x_{1} /\left.\partial c\right|_{c=0}$ is negative for $\kappa>1 / \sqrt{2}$. Hence, $x_{1}$ is a decreasing function of $c$ around $c=0$. As a consequence, for $\kappa>1 / \sqrt{2}$, adding a small capacitance in parallel to the inductance increases the ETDR, 


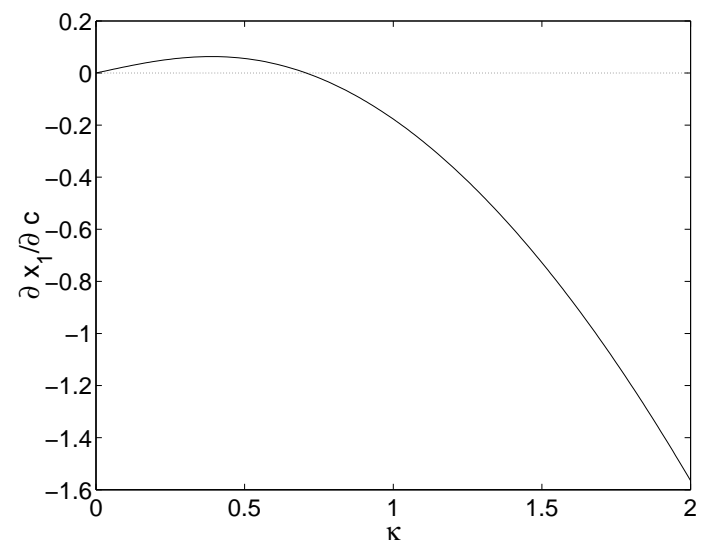

Fig. 2. Behaviour of $\partial x_{1} / \partial c$ versus $\kappa$ for $c=0$

proving that the proposed shunt circuit performs better than the classical $R L$ one. It is emphasized that this conclusion is not limited by the assumed factorization (11) used in the computations. Indeed, if another factorization of $p(S)$ exist yielding a higher ETDR than the one supplied by (11), a-fortiori that ETDR would be better than the one provided by the $R L$ shunt circuit.

\subsection{Optimization of the $R(L \| C)$ shunt circuit}

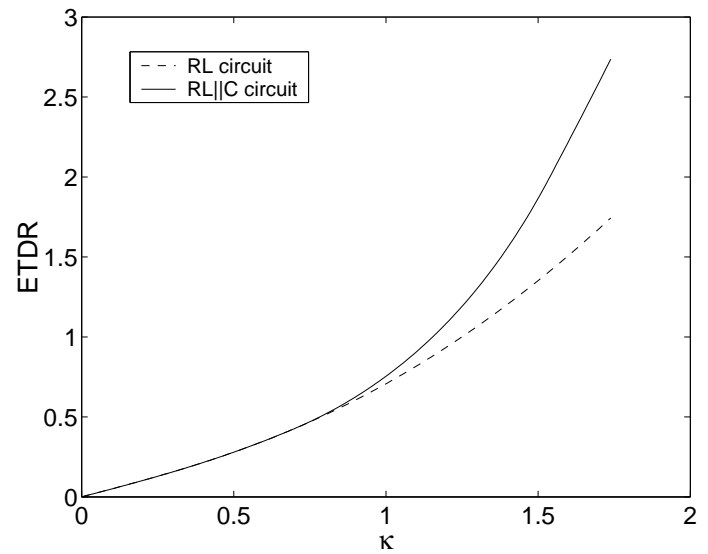

Fig. 3. Behavior of ETDR versus $\kappa$

In this section an analytical optimization of the proposed circuit is performed. In particular, the optimal values of $\rho, \lambda$ and $c$, and the corresponding 


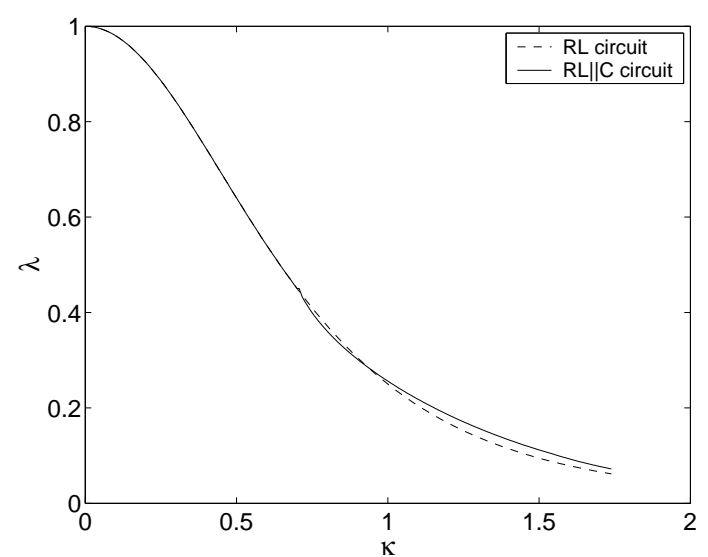

Fig. 4. Behavior of $\lambda$ versus $\kappa$

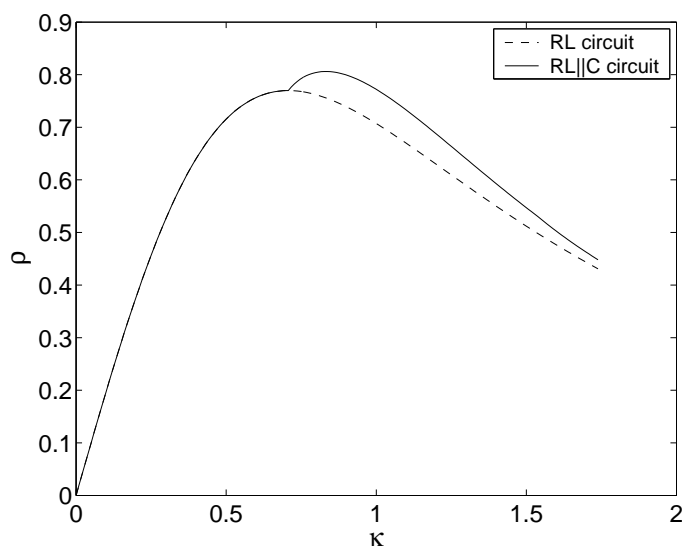

Fig. 5. Behavior of $\rho$ versus $\kappa$

ETDR, are determined for given values of the piezoelectric coupling coefficient $\kappa$.

As a consequence of equation (12), it turns out that, for $\kappa \leq 1 / \sqrt{2}$ no increase in performances can be obtained with respect to the $R L$ circuit if a small capacitance $C$ is added in parallel to the inductance $L$. Accordingly, in this range the optimal value of $c$, near $c=0$, is just zero, and the optimal expressions for the other parameters are those relevant to the $R L$ circuit, given in equation (10).

When $\kappa>1 / \sqrt{2}$, equation (12) implies that there exists an optimal value of $c$, strictly greater than zero, yielding the optimal ETDR. In order to apply the pole placement technique to find the optimum, a special factorization of $p(S)$ is needed. This factorization is suggested by the case of the resistive-inductive shunt circuit. In that case, two coincident couples of com- 


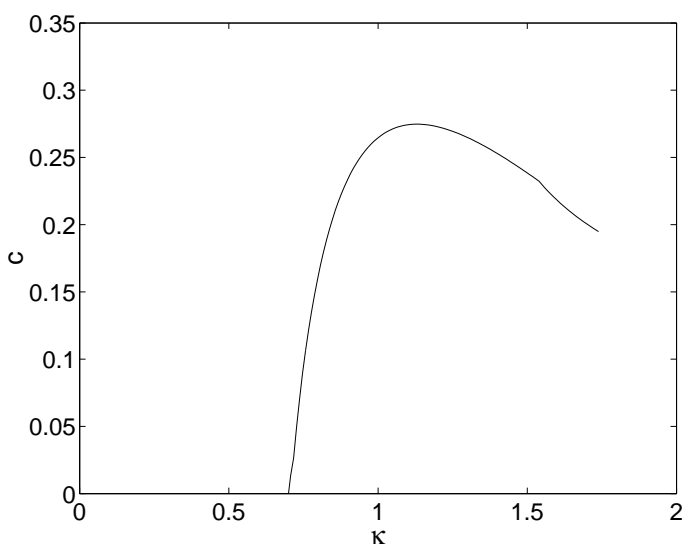

Fig. 6. Behaviour of $c$ versus $\kappa$

plex conjugate roots can be collocated. In the $R(L \| C)$ case, the characteristic polynomial $p(S)$ is fifth-order degree. Hence, an extra real root exists and, accordingly, the factorization (11) is considered. By identifying the expressions (6) and (11) of $p(S)$, five nonlinear equations can be written involving the six unknowns $x_{1}, x_{2}, x_{3}, \rho, \lambda$ and $c$. These equations define implicitly $x_{1}$, $x_{2}, x_{3}, \rho$ and $\lambda$ as functions of $c$. Thus, the extra condition of the stationarity of $x_{1}=-$ ETDR with respect to $c$ can be added. In the range $\kappa>1 / \sqrt{2}$ an admissible solution to this stationary condition can be found and is reported in Appendix 4.1.

For $\kappa=\bar{\kappa} \simeq 1.53$, computed in Appendix 4.1, it turns out that $x_{1}=$ $x_{3}$, so that all the real parts of the poles are coincident. When $\kappa>\bar{\kappa}$ the factorization (11) is no longer optimal, since $x_{3}$ would became greater than $x_{1}$. Hence, the optimal factorization enforces that the double complex poles and the real pole have the same real part:

$$
p(S)=\left(S^{2}-2 x_{1} S+x_{1}^{2}+x_{2}^{2}\right)^{2}\left(S-x_{1}\right)
$$

In this case, there are only five unknowns $x_{1}, x_{2}, \rho, \lambda$ and $c$ involved into the five nonlinear equations coming from the polynomial identification, which determine the admissible solution reported in Appendix 4.2.

Finally, for $\kappa=\tilde{\kappa} \simeq 1.75$, computed in Appendix 4.2, there exists a choice of the electrical parameters such that the polynomial $p(S)$ exhibits a fifthorder real pole, and the best possible ETDR supplied by the proposed shunt circuit is attained.

The values of the ETDR, $\lambda, \rho$ and $c$ are respectively plotted in Figs. (3)(6) versus the piezoelectric coupling coefficient $\kappa$, and compared to the corresponding quantities relevant to the $R L$ circuit. From Fig. 3 it turns out that, for $\kappa>1 / \sqrt{2}$, the $R(L \| C)$ circuit exhibits a greater ETDR than the classical $R L$ circuit. Moreover the difference between the performances of the 
two shunt circuits increases with increasing $\kappa$. From Figs. 4 and 5 it follows that the optimal inductance and resistance relevant to the $R(L \| C)$ circuit are almost the same as the ones required by the $R L$ shunt circuit. Finally, Fig. 6 shows that $c$ has a maximum of the order of 0.3 ; hence, the capacitance $C$ to be put in parallel to the inductance $L$ is less than $30 \%$ of the piezoelectric capacity $C_{p}$.

The above results show that the proposed shunt circuit performs better than the $R L$ shunt circuit for $\kappa>1 / \sqrt{2}$, and requires values of the electrical component very close to the ones required by the $R L$ circuit.

\section{Conclusions}

In this paper the problem of choosing the optimal shunt circuit for singlemode passive vibration damping was discussed. It was shown that, at least for sufficiently high coupling coefficients $\kappa$, the traditional $R L$ circuit is not the most effective. Indeed, the performance of a new shunt circuit proposed here is definitely better. It is emphasized that in modern applications very high values of $\kappa$ can be achieved, for instance by using negative capacitances. In multi-modal vibration damping the basic design concepts remain the same and therefore the problem of choosing the optimal shunt circuit must be faced also in that case.

\section{Acknowledgments}

This research was developed within the framework of Lagrange Laboratory, an European research group between CNRS, CNR, University of Rome "Tor Vergata", University of Montpellier II, ENPC and LCPC.

\section{References}

1. Caruso, G. (2000) Laminati piezoelettrici, modellazione, algoritmi di calcolo ed ottimizzazione della risposta dinamica. Doctoral Thesis, Dep. of Civil Engineering, University of Rome "Tor Vergata".

2. Agnes, G. S. (1995) Development of a modal model for simultaneous active and passive piezoelectric vibration suppression. J. Intell. Mater. Syst. Struct. 6, 482-487

3. Edberg, D. L., Bicos, A. S., and Fechter, J. S. (1991) On piezoelectric energy conversion for electronic passive damping enhancement. Proc. Damping '91 (San Diego, CA, 1991) paper GBA-1.

4. Bisegna, P., Caruso, G., Del Vescovo D., Galeani, S. and Menini, L. (2000) Semiactive control of a thin piezoactuated structure. SPIE Proc. 3989, 300-311

5. Inman, D. J. (1997) Vibration suppression trough smart damping. Proc. of the Fifth International Congress on Sound and Vibration, Adelaide, South Australia

6. Lesieutre, G. A. (1998) Vibration damping and control using shunted piezoelectric materials. Shock vibr. digest 30, 181-190 
7. Hagood, N. W. and von Flotow, A. (1991) Damping of structural vibrations with piezoelectric materials and passive electrical networks. J. Sound Vib. 146, $243-268$

8. Wu, S. Y. (1996) Piezoelectric shunts with a parallel R-L circuit for structural damping and vibration control. SPIE Proc. 2720 259-269

9. Park, C. H. and Inman, D. J. (1999) A uniform model for series R-L and parallel R-L shunt circuits and power consumption. SPIE Proc. 3668 797-804

10. Park, C. H. (2003) Dynamics modelling of beams with shunted piezoelectric elements. J. Sound Vib. 268 115-129

11. Park, C. H., Kabeya, K. and Inman, D. J. (1998) Enhanced piezoelectric shunt design. Trans. ASME 57 149-155

12. Caruso, G. (2000) Smorzmento passivo di vibrazioni mediante materiali piezoelettrici e circuiti elettrici risonanti. Proc. XXIX National Congress AIAS, Lucca, Italy, 307-316

13. Caruso, G. (2001) A critical analysis of electric shunt circuits employed in piezoelectric passive vibration damping. Smart Mater. Struct. 10 1059-1068

14. Tang, J. and Wang, K. W. (2001) Active-passive hybrid piezoelectric networks for vibration control: comparisons and improvement. Smart Mater. Struct. 10 794-806.

15. Bisegna, P. and Caruso, G. (2000) Mindlin-type finite elements for piezoelectric sandwich plates J. Intell. Mater. Syst. Struct. 11 14-25

\section{Appendix}

\subsection{Optimization according to factorization (11) with stationary $x_{1}$}

For $1 / \sqrt{2}<\kappa<\bar{\kappa}$ the optimal value of $x_{1}$ is given by

$$
x_{1}=-\sqrt{\max _{i}\left\{z_{i}\right\}}
$$

where $z_{i}$ are the positive real roots of the polynomial in the unknown $z$

$$
\begin{aligned}
& q(z)=4096\left(\kappa^{2}-8\right)^{2} z^{8} \\
& +2048\left(-7 \kappa^{6}+42 \kappa^{4}-336 \kappa^{2}+128\right) z^{7} \\
& \quad+512\left(36 \kappa^{8}+91 \kappa^{6}+635 \kappa^{4}+448 \kappa^{2}+192\right) z^{6} \\
& + \\
& \quad+16\left(\kappa^{12}-644 \kappa^{10}-5324 \kappa^{8}-9720 \kappa^{6}-5520 \kappa^{4}+896 \kappa^{2}+1024\right) z^{5} \\
& \quad-8\left(1+\kappa^{2}\right)\left(7 \kappa^{12}-230 \kappa^{10}-1210 \kappa^{8}+2270 \kappa^{6}\right. \\
& \left.\quad+6238 \kappa^{4}+2912 \kappa^{2}-128\right) z^{4} \\
& +\kappa^{2}\left(1+\kappa^{2}\right)^{2}\left(73 \kappa^{10}+684 \kappa^{8}+6964 \kappa^{6}+14544 \kappa^{4}+8096 \kappa^{2}-256\right) z^{3} \\
& \quad-\kappa^{4}\left(1+\kappa^{2}\right)^{3}\left(43 \kappa^{8}+488 \kappa^{6}+1596 \kappa^{4}+1024 \kappa^{2}-16\right) z^{2} \\
& +\kappa^{8}\left(1+\kappa^{2}\right)^{4}\left(11 \kappa^{4}+76 \kappa^{2}+44\right) z \\
& -\kappa^{12}\left(1+\kappa^{2}\right)^{5}
\end{aligned}
$$

The optimal values of the electrical parameters are given by

$$
\lambda=-\frac{\left(1+\kappa^{2}-4 x_{1} x_{3}-4 x_{1}^{2}-2 y\right) y^{2} x_{3}}{\Lambda}
$$




$$
\begin{aligned}
& c=\frac{\lambda}{\left(4 x_{1} x_{3}+4 x_{1}^{2}+2 y-1-\kappa^{2}\right)} \\
& \rho=\frac{(1+c)}{-c\left(x_{3}+4 x_{1}\right)}
\end{aligned}
$$

where

$$
\begin{aligned}
& x_{3}=-\frac{1}{4} \frac{y^{2}-2 y-2 \kappa^{2} y-4 x_{1}^{2} \kappa^{2}+2 \kappa^{2}-4 x_{1}^{2}+1+\kappa^{4}}{x_{1}\left(-1+y-\kappa^{2}\right)} \\
& \Lambda=y^{2} x_{3}-4 x_{3}^{2} x_{1}-20 x_{1}^{2} x_{3}-2 y x_{3}+x_{3}+x_{3} \kappa^{2}-16 x_{1}^{3}-8 y x_{1}+4 x_{1}+4 x_{1} \kappa^{2}(20)
\end{aligned}
$$

and $y$ is given by the maximum positive real root of the following polynomial in the unknown $y$

$$
\begin{gathered}
r(y)=y^{5}+\left(4 x_{1}^{2}-4 \kappa^{2}-5\right) y^{4}+\left(10+16 \kappa^{2}-12 x_{1}^{2} \kappa^{2}-8 x_{1}^{2}+6 \kappa^{4}\right) y^{3} \\
+\left(32 x_{1}^{4}-10-4 \kappa^{6}-16 x_{1}^{4} \kappa^{2}+12 x_{1}^{2} \kappa^{2}-18 \kappa^{4}-24 \kappa^{2}+12 x_{1}^{2} \kappa^{4}\right) y^{2} \\
+\left(5+8 x_{1}^{2}-4 \kappa^{6} x_{1}^{2}+18 \kappa^{4}+12 x_{1}^{2} \kappa^{2}-48 x_{1}^{4}+16 \kappa^{2}\right. \\
\left.+\kappa^{8}-48 x_{1}^{4} \kappa^{2}+8 \kappa^{6}\right) y \\
-1 / 4-3 x_{1}^{2} \kappa^{2}-3 \kappa^{4} / 2-\kappa^{6}-\kappa^{2}+4 x_{1}^{4}-3 x_{1}^{2} \kappa^{4} \\
+4 \kappa^{4} x_{1}^{4}-\kappa^{8} / 4-x_{1}^{2}+16 x_{1}^{6} \kappa^{2}+16 x_{1}^{6}+8 x_{1}^{4} \kappa^{2}-\kappa^{6} x_{1}^{2}
\end{gathered}
$$

The imaginary part of the double complex conjugate roots is $x_{2}= \pm \sqrt{y-x_{1}^{2}}$.

The present factorization supplies an optimal ETDR as long as the real root $x_{3}$ is less than or equal to the real part $x_{1}$ of the double complex conjugate roots. The limit $\bar{\kappa}$ in Section 3.2 is just defined as the value of $\kappa$ which makes the equality prevail. It is given by the real positive solution of the following system in the unknowns $\kappa$ and $x_{1}$

$$
\begin{aligned}
& A\left(4 x_{1}^{2} \kappa^{2}-2+\kappa^{4}-\kappa^{2}-2 x_{1}^{2}\right) \\
& \quad=6 x_{1}^{2}+6 x_{1}^{4}+\kappa^{2} x_{1}^{2}-5 \kappa^{4} x_{1}^{2}+4 \kappa^{2} x_{1}^{4} \\
& \begin{array}{l}
A\left(6 x_{1}^{2}+13 \kappa^{2}+9 \kappa^{6}-32 x_{1}^{4}+20 \kappa^{4}-8 x_{1}^{4} \kappa^{2}+2+16 x_{1}^{2} \kappa^{4}+22 x_{1}^{2} \kappa^{2}\right) \\
\quad=-8 \kappa^{2} x_{1}^{6}-3 \kappa^{6}-62 \kappa^{4} x_{1}^{2}+34 \kappa^{2} x_{1}^{4}+32 x_{1}^{6}-26 \kappa^{6} x_{1}^{2}-\kappa^{8} \\
\quad+22 x_{1}^{4}-10 x_{1}^{2}-\kappa^{2}+12 \kappa^{4} x_{1}^{4}-3 \kappa^{4}-46 \kappa^{2} x_{1}^{2}
\end{array}
\end{aligned}
$$

where $A=\sqrt{x_{1}^{2}\left(1+x_{1}^{2}+\kappa^{2}\right)}$.

\subsection{Optimization according to factorization (13)}

For $\bar{\kappa}<\kappa<\tilde{\kappa}$ the optimal value of $x_{1}$ is given by

$$
x_{1}=-\frac{\sqrt{2}}{2} \sqrt{\frac{2 \kappa^{6}-3 \kappa^{2}+\kappa^{4}-2+\sqrt{4 \kappa^{12}-8 \kappa^{8}+2 \kappa^{10}-6 \kappa^{6}}}{1+2 \kappa^{2}}}
$$


and the optimal parameters are

$$
\begin{aligned}
\lambda & =\frac{\left(8 x_{1}^{2}+2 y-1-\kappa^{2}\right) y^{2}}{-y^{2}+40 x_{1}^{2}+10 y-5-5 \kappa^{2}} \\
c & =\frac{\lambda}{8 x_{1}^{2}+2 y-1-\kappa^{2}} \\
\rho & =\frac{1}{5} \frac{1+c}{\left(-x_{1}\right) c}
\end{aligned}
$$

where

$$
y=-2 x_{1}^{2}+\kappa^{2}+1+2 \sqrt{x_{1}^{4}+\kappa^{2} x_{1}^{2}+x_{1}^{2}}
$$

The present factorization is admissible as long as two double complex conjugate roots exist. When $\kappa \rightarrow \tilde{\kappa}$, given by

$$
\tilde{\kappa}=\sqrt{24+6 \sqrt{5}-\frac{2}{25} \sqrt{5}(125+30 \sqrt{5})}
$$

the imaginary part $x_{2}= \pm \sqrt{y-x_{1}^{2}}$ of those conjugate roots tends to zero, and for $\kappa=\tilde{\kappa}$ the characteristic polynomial $p(S)$ admits five coincident real roots. 